\title{
Electronic Structure Control of the Nucleophilicity of Transition Metal-Thiolate Complexes: An Experimental and Theoretical Study.
}

Derek C. Fox, ${ }^{\dagger}$ Adam T. Fiedler, ${ }^{\dagger}$ Heather L. Halfen, ${ }^{\dagger}$ Thomas C. Brunold, ${ }^{*}{ }^{\star}$ and Jason A. Halfen ${ }^{*} \dagger$

Contribution from the University of Wisconsin, Department of Chemistry, $1101 \mathrm{~W}$. University Avenue, Madison, WI 53706, and the University of Wisconsin-Eau Claire, Department of Chemistry, 105 Garfield Avenue, Eau Claire, WI 54702.

${ }^{\dagger}$ University of Wisconsin-Eau Claire

¿niversity of Wisconsin 


\section{Table of contents}

\section{$\underline{\text { Page }}$}

Table S1

Table S2

Table S3

Table S4

Figure S1

Figure S2

Figure S3

Figure S4
S3

S3

S3

S4-S11

$\mathrm{S} 12$

S12

S13

S13 
Table S1. Pseudo-first order rate constants $\left(k_{\mathrm{obs}}\right)$ for the reactions of $\mathbf{1}, \mathbf{2}$, and $\mathbf{3}$ with benzyl bromide. ${ }^{a}$

\begin{tabular}{|c|l|l|l|}
\hline Complex & \multicolumn{3}{|c|}{$k_{\mathrm{obs}}, \mathrm{s}^{-1}$} \\
\hline $\mathbf{1}$ & $5.0(3)^{*} 10^{-4}$ & $5.3(3)^{*} 10^{-4}$ & $5.2(3)^{*} 10^{-4}$ \\
\hline $\mathbf{2}$ & $3.49(9)^{*} 10^{-4}$ & $3.40(8)^{*} 10^{-4}$ & $3.49(7)^{*} 10^{-4}$ \\
\hline $\mathbf{3}$ & $3.56(4)^{*} 10^{-3}$ & $3.48(7)^{*} 10^{-3}$ & $3.60(8)^{*} 10^{-3}$ \\
\hline
\end{tabular}

${ }^{a}$ Rate constants determined at 297(1) K with $0.50 \mathrm{mM}$ complex, $172 \mathrm{mM}$ benzyl bromide in $\mathrm{CH}_{3} \mathrm{CN}$.

Table S2. Pseudo-first order rate constants $\left(k_{\mathrm{obs}}\right)$ for the reaction of $\mathbf{4}$ with benzyl bromide. ${ }^{a}$

\begin{tabular}{|c|l|l|l|}
\hline Complex & \multicolumn{3}{|c|}{$k_{\mathrm{obs}}, \mathrm{s}^{-1}$} \\
\hline $\mathbf{4}$ & $2.4(2)^{*} 10^{-3}$ & $2.6(3)^{*} 10^{-3}$ & $2.4(3)^{*} 10^{-3}$ \\
\hline
\end{tabular}

${ }^{a}$ Rate constants determined at $303 \mathrm{~K}$ with $5.0 \mathrm{mM}$ complex, $86 \mathrm{mM}$ benzyl bromide in $\mathrm{CD}_{3} \mathrm{CN}$.

Table S3. Pseudo-first order rate constants $\left(k_{\mathrm{obs}}, \mathrm{s}^{-1}\right)$ for the reactions of $\mathbf{2}$ and $\mathbf{3}$ with benzyl bromide as a function of temperature.

\begin{tabular}{|c|c|c|c|c|c|c|}
\hline Complex & $294 \mathrm{~K}$ & $297 \mathrm{~K}$ & $303 \mathrm{~K}$ & $310 \mathrm{~K}$ & $316 \mathrm{~K}$ & $318 \mathrm{~K}$ \\
\hline \multirow{3}{*}{$2^{a}$} & & $3.49(9)^{*} 10^{-4}$ & $5.52(6) * 10^{-4}$ & $8.10(6)^{*} 10^{-4}$ & & $1.28(1)^{*} 10^{-3}$ \\
\cline { 2 - 7 } & & $3.40(8)^{*} 10^{-4}$ & $5.58(4)^{*} 10^{-4}$ & $8.27(4)^{*} 10^{-4}$ & & $1.26(1)^{*} 10^{-3}$ \\
\cline { 2 - 7 } & & $3.49(7)^{*} 10^{-4}$ & $5.11(2)^{*} 10^{-4}$ & & & $1.26(1)^{*} 10^{-3}$ \\
\hline \multirow{3}{*}{$3^{b}$} & $1.45(4)^{*} 10^{-3}$ & & $2.60(1)^{*} 10^{-3}$ & $3.98(2)^{*} 10^{-3}$ & $5.17(4)^{*} 10^{-3}$ & \\
\cline { 2 - 7 } & $1.44(7)^{*} 10^{-3}$ & & $2.52(1)^{*} 10^{-3}$ & $4.08(2)^{*} 10^{-3}$ & $5.12(3) * 10^{-3}$ & \\
\cline { 2 - 7 } & $1.52(8) * 10^{-3}$ & & $2.50(1)^{*} 10^{-3}$ & & & \\
\hline
\end{tabular}

${ }^{a}$ Reactions conducted with $\left[\mathrm{PhCH}_{2} \mathrm{Br}\right]=172 \mathrm{mM} .{ }^{b}$ Reactions conducted with $\left[\mathrm{PhCH}_{2} \mathrm{Br}\right]=86$ $\mathrm{mM}$ 
Table S4. Cartesian coordinates for DFT-optimized structures.

\section{$\left[\mathrm{L}^{8} \mathrm{py}_{2} \mathrm{Co}\left(\mathrm{S}-\mathrm{C}_{6} \mathrm{H}_{4}-p-\mathrm{CH}_{3}\right)\right]^{+}(2)$}

\begin{tabular}{|c|c|c|c|}
\hline Co & 0.000000 & 0.000000 & 0.000000 \\
\hline S & 0.000000 & 0.000000 & 2.223557 \\
\hline C & -1.529968 & -0.814560 & 2.667297 \\
\hline C & -2.651581 & -0.065170 & 3.056763 \\
\hline C & -3.858124 & -0.700409 & 3.358963 \\
\hline $\mathrm{C}$ & -3.987700 & -2.096359 & 3.293671 \\
\hline C & -2.849472 & -2.840973 & 2.936264 \\
\hline $\mathrm{C}$ & -1.639420 & -2.216995 & 2.631210 \\
\hline $\mathrm{C}$ & -5.295746 & -2.780380 & 3.614380 \\
\hline $\mathrm{H}$ & -2.576340 & 1.020859 & 3.118790 \\
\hline $\mathrm{H}$ & -4.719208 & -0.095657 & 3.651413 \\
\hline $\mathrm{H}$ & -2.908508 & -3.931580 & 2.902908 \\
\hline $\mathrm{H}$ & -0.767227 & -2.816742 & 2.368225 \\
\hline $\mathrm{H}$ & -5.218414 & -3.373245 & 4.538010 \\
\hline $\mathrm{H}$ & -5.593262 & -3.473007 & 2.813370 \\
\hline $\mathrm{H}$ & -6.104950 & -2.052444 & 3.751968 \\
\hline $\mathrm{N}$ & 0.200211 & 2.062149 & -0.677429 \\
\hline $\mathrm{N}$ & 2.228348 & -0.006287 & -0.310 \\
\hline $\mathrm{N}$ & -2.017685 & 0.357925 & -0.51 \\
\hline $\mathrm{N}$ & 0.397797 & -1.984924 & -0.721039 \\
\hline $\mathrm{C}$ & 1.115555 & 2.848083 & 0.212 \\
\hline C & 2.611328 & 2.454620 & 0.153259 \\
\hline C & 2.931870 & 0.991028 & 0.539 \\
\hline C & 2.531494 & 0.213257 & -1.764923 \\
\hline C & 1.323166 & 0.759033 & -2.585297 \\
\hline C & 0.651978 & 2.080154 & -2.114044 \\
\hline C & -1.158188 & 2.644669 & -0.534866 \\
\hline $\mathrm{C}$ & -2.278503 & 1.659698 & -0.786850 \\
\hline C & -3.554749 & 2.108002 & -1.141052 \\
\hline C & -4.609940 & 1.197617 & -1.187302 \\
\hline C & -4.354080 & -0.137573 & -0.865906 \\
\hline $\mathrm{C}$ & -3.053802 & -0.510437 & -0.538864 \\
\hline C & 2.599152 & -1.369202 & 0.120667 \\
\hline $\mathrm{C}$ & 1.664795 & -2.397278 & -0.471405 \\
\hline $\mathrm{C}$ & 2.079208 & -3.708755 & -0.719528 \\
\hline C & 1.167389 & -4.628891 & -1.236053 \\
\hline $\mathrm{C}$ & -0.132370 & -4.200714 & -1.515121 \\
\hline $\mathrm{C}$ & -0.468262 & -2.876343 & -1.250717 \\
\hline $\mathrm{H}$ & 0.744492 & 2.712189 & 1.239334 \\
\hline $\mathrm{H}$ & 1.034317 & 3.922211 & -0.046860 \\
\hline $\mathrm{H}$ & 3.140671 & 3.106598 & 0.866500 \\
\hline $\mathrm{H}$ & 3.033890 & 2.692795 & -0.835373 \\
\hline $\mathrm{H}$ & 4.029083 & 0.840805 & 0.471893 \\
\hline $\mathrm{H}$ & 2.630676 & 0.803116 & 1.582672 \\
\hline $\mathrm{H}$ & 2.813553 & -0.750931 & -2.215775 \\
\hline $\mathrm{H}$ & 3.407684 & 0.874924 & -1.870056 \\
\hline $\mathrm{H}$ & 0.549149 & -0.025970 & -2.623215 \\
\hline $\mathrm{H}$ & 1.661300 & 0.912857 & -3.624344 \\
\hline $\mathrm{H}$ & 1.317322 & 2.946945 & -2.277237 \\
\hline $\mathrm{H}$ & -0.236023 & 2.235260 & -2.746582 \\
\hline $\mathrm{H}$ & -1.279419 & 3.542984 & -1.163437 \\
\hline $\mathrm{H}$ & -1.258163 & 2.970734 & 0.512115 \\
\hline
\end{tabular}




$\begin{array}{rrrr}\mathrm{H} & -3.715286 & 3.164200 & -1.362686 \\ \mathrm{H} & -5.614243 & 1.524643 & -1.458115 \\ \mathrm{H} & -5.147995 & -0.883804 & -0.861374 \\ \mathrm{H} & -2.824158 & -1.535721 & -0.251831 \\ \mathrm{H} & 2.502594 & -1.402481 & 1.218353 \\ \mathrm{H} & 3.645981 & -1.616500 & -0.134613 \\ \mathrm{H} & 3.110092 & -3.998535 & -0.509857 \\ \mathrm{H} & 1.470337 & -5.658249 & -1.430954 \\ \mathrm{H} & -0.874680 & -4.876633 & -1.938919 \\ \mathrm{H} & -1.463272 & -2.499405 & -1.479050\end{array}$

\section{$\left[\mathrm{L}^{8} \mathrm{py}_{2} \mathrm{Ni}\left(\mathrm{S}-\mathrm{C}_{6} \mathrm{H}_{4}-p-\mathrm{CH}_{3}\right)\right]^{+}(\mathbf{3})$}

$\begin{array}{lrrr}\mathrm{Ni} & 0.000000 & 0.000000 & 0.000000 \\ \mathrm{~S} & 0.000000 & 0.000000 & 2.334961 \\ \mathrm{C} & 1.278549 & 1.142349 & 2.794388 \\ \mathrm{C} & 2.537933 & 0.675247 & 3.216141 \\ \mathrm{C} & 3.557755 & 1.568283 & 3.548996 \\ \mathrm{C} & 3.366455 & 2.957291 & 3.474869 \\ \mathrm{C} & 2.097855 & 3.421967 & 3.078217 \\ \mathrm{C} & 1.071655 & 2.537796 & 2.751160 \\ \mathrm{C} & 4.471954 & 3.924240 & 3.824539 \\ \mathrm{H} & 2.707306 & -0.399948 & 3.281952 \\ \mathrm{H} & 4.525146 & 1.177872 & 3.872818 \\ \mathrm{H} & 1.908920 & 4.497681 & 3.038162 \\ \mathrm{H} & 0.090942 & 2.919510 & 2.464661 \\ \mathrm{H} & 4.664978 & 4.627700 & 3.000732 \\ \mathrm{H} & 5.408417 & 3.399033 & 4.049072 \\ \mathrm{H} & 4.206711 & 4.529175 & 4.704300 \\ \mathrm{~N} & 0.194931 & -2.087173 & -0.476151 \\ \mathrm{~N} & -2.120682 & -0.329529 & -0.221695 \\ \mathrm{~N} & 2.033371 & -0.017441 & -0.310257 \\ \mathrm{~N} & -0.634293 & 1.954697 & -0.542557 \\ \mathrm{C} & -0.604431 & -2.981155 & 0.417068 \\ \mathrm{C} & -2.137085 & -2.820129 & 0.299362 \\ \mathrm{C} & -2.670624 & -1.412064 & 0.643921 \\ \mathrm{C} & -2.357178 & -0.618256 & -1.676773 \\ \mathrm{C} & -1.058014 & -1.010590 & -2.449127 \\ \mathrm{C} & -0.210388 & -2.209030 & -1.921875 \\ \mathrm{C} & 1.625015 & -2.418747 & -0.278091 \\ \mathrm{C} & 2.550552 & -1.251404 & -0.522278 \\ \mathrm{C} & 3.903641 & -1.451553 & -0.805328 \\ \mathrm{C} & 4.765579 & -0.355240 & -0.835953 \\ \mathrm{C} & 4.245865 & 0.910446 & -0.560471 \\ \mathrm{C} & 2.883850 & 1.032410 & -0.301331 \\ \mathrm{C} & -2.719177 & 0.956284 & 0.206711 \\ \mathrm{C} & -1.967117 & 2.130341 & -0.363556 \\ \mathrm{C} & -2.604858 & 3.338776 & -0.655197 \\ \mathrm{C} & -1.856522 & 4.406509 & -1.150085 \\ \mathrm{C} & -0.489563 & 4.220581 & -1.363785 \\ \mathrm{C} & 0.072739 & 2.985474 & -1.054184 \\ \mathrm{H} & -0.291977 & -2.748367 & 1.447403 \\ \mathrm{H} & -0.346085 & -4.036575 & 0.201889 \\ \mathrm{H} & -2.591751 & -3.524841 & 1.013794 \\ \mathrm{H} & -2.488388 & -3.137177 & -0.695160 \\ \mathrm{H} & -3.776047 & -1.419510 & 0.562546 \\ & & & \end{array}$

- S5 - 


$\begin{array}{rrrr}\mathrm{H} & -2.408981 & -1.155121 & 1.682907 \\ \mathrm{H} & -2.750397 & 0.292023 & -2.155000 \\ \mathrm{H} & -3.132721 & -1.394608 & -1.785019 \\ \mathrm{H} & -0.404144 & -0.121307 & -2.493042 \\ \mathrm{H} & -1.338348 & -1.237259 & -3.492081 \\ \mathrm{H} & -0.735840 & -3.167786 & -2.077362 \\ \mathrm{H} & 0.707000 & -2.244064 & -2.529953 \\ \mathrm{H} & 1.925629 & -3.295227 & -0.876100 \\ \mathrm{H} & 1.744980 & -2.692841 & 0.781509 \\ \mathrm{H} & 4.275986 & -2.462112 & -0.979630 \\ \mathrm{H} & 5.826218 & -0.490402 & -1.049881 \\ \mathrm{H} & 4.882141 & 1.794449 & -0.535477 \\ \mathrm{H} & 2.451630 & 1.996277 & -0.039948 \\ \mathrm{H} & -2.628571 & 0.992752 & 1.305008 \\ \mathrm{H} & -3.789734 & 1.017120 & -0.055405 \\ \mathrm{H} & -3.679977 & 3.434158 & -0.496017 \\ \mathrm{H} & -2.334198 & 5.359741 & -1.378998 \\ \mathrm{H} & 0.135880 & 5.015167 & -1.770157 \\ \mathrm{H} & 1.129837 & 2.803223 & -1.233810\end{array}$

\section{$\left[\mathrm{L}^{8} \mathrm{py}_{2} \mathrm{Zn}\left(\mathrm{S}-\mathrm{C}_{6} \mathrm{H}_{4}-p-\mathrm{CH}_{3}\right)\right]^{+}$(4)}

$\begin{array}{lrrr}\text { Zn } & 0.000000 & 0.000000 & 0.000000 \\ \text { S } & 0.000000 & 0.000000 & 2.288208 \\ \text { C } & 1.365631 & 1.150314 & 2.547348 \\ \text { C } & 2.652985 & 0.675797 & 2.837997 \\ \text { C } & 3.712280 & 1.566818 & 3.039520 \\ \text { C } & 3.527008 & 2.954758 & 2.964203 \\ \text { C } & 2.228700 & 3.422516 & 2.695572 \\ \text { C } & 1.164490 & 2.540741 & 2.495819 \\ \mathrm{C} & 4.669891 & 3.916855 & 3.192154 \\ \mathrm{H} & 2.823029 & -0.399368 & 2.908386 \\ \mathrm{H} & 4.705231 & 1.169891 & 3.263153 \\ \mathrm{H} & 2.040955 & 4.498535 & 2.657684 \\ \mathrm{H} & 0.161407 & 2.928452 & 2.312912 \\ \mathrm{H} & 4.774551 & 4.626404 & 2.358154 \\ \mathrm{H} & 5.622879 & 3.385895 & 3.307373 \\ \mathrm{H} & 4.509171 & 4.514328 & 4.101500 \\ \mathrm{~N} & 0.160248 & -2.096512 & -0.868698 \\ \mathrm{~N} & -2.184219 & -0.362671 & -0.529953 \\ \mathrm{~N} & 2.046844 & -0.028549 & -0.704636 \\ \mathrm{~N} & -0.715775 & 1.936447 & -0.879089 \\ \mathrm{C} & -0.624405 & -3.018295 & 0.013000 \\ \mathrm{C} & -2.163086 & -2.862534 & -0.069458 \\ \mathrm{C} & -2.720169 & -1.469666 & 0.312057 \\ \mathrm{C} & -2.412247 & -0.617813 & -1.990936 \\ \mathrm{C} & -1.114197 & -0.954605 & -2.780151 \\ \mathrm{C} & -0.268005 & -2.168335 & -2.307907 \\ \mathrm{C} & 1.591125 & -2.432861 & -0.717926 \\ \mathrm{C} & 2.523682 & -1.264618 & -0.964523 \\ \mathrm{C} & 3.860764 & -1.480377 & -1.309219 \\ \mathrm{C} & 4.737930 & -0.397415 & -1.354294 \\ \mathrm{C} & 4.254044 & 0.873627 & -1.041168 \\ \mathrm{C} & 2.907455 & 1.011810 & -0.720322 \\ \mathrm{C} & -2.787994 & 0.915131 & -0.096680 \\ \mathrm{C} & -2.042206 & 2.099945 & -0.670212 \\ & & & \end{array}$

- S6 - 


$\begin{array}{lrrr}\mathrm{C} & -2.690308 & 3.309265 & -0.941711 \\ \mathrm{C} & -1.955276 & 4.379730 & -1.449158 \\ \mathrm{C} & -0.591797 & 4.201981 & -1.695206 \\ \mathrm{C} & -0.018738 & 2.969482 & -1.397095 \\ \mathrm{H} & -0.294281 & -2.821136 & 1.045929 \\ \mathrm{H} & -0.371979 & -4.068100 & -0.235200 \\ \mathrm{H} & -2.595474 & -3.589676 & 0.636520 \\ \mathrm{H} & -2.527863 & -3.163055 & -1.064178 \\ \mathrm{H} & -3.825897 & -1.498428 & 0.235229 \\ \mathrm{H} & -2.463318 & -1.236298 & 1.358414 \\ \mathrm{H} & -2.839523 & 0.289917 & -2.444672 \\ \mathrm{H} & -3.166200 & -1.413284 & -2.116714 \\ \mathrm{H} & -0.467911 & -0.061493 & -2.776900 \\ \mathrm{H} & -1.395630 & -1.134583 & -3.831390 \\ \mathrm{H} & -0.802017 & -3.119339 & -2.485031 \\ \mathrm{H} & 0.638138 & -2.194717 & -2.933563 \\ \mathrm{H} & 1.877869 & -3.291275 & -1.348679 \\ \mathrm{H} & 1.747116 & -2.739136 & 0.328705 \\ \mathrm{H} & 4.209183 & -2.492676 & -1.518723 \\ \mathrm{H} & 5.787109 & -0.547058 & -1.610733 \\ \mathrm{H} & 4.906204 & 1.746155 & -1.032913 \\ \mathrm{H} & 2.500061 & 1.979950 & -0.433395 \\ \mathrm{H} & -2.709900 & 0.956696 & 1.003143 \\ \mathrm{H} & -3.857773 & 0.977707 & -0.364227 \\ \mathrm{H} & -3.763138 & 3.399170 & -0.764740 \\ \mathrm{H} & -2.443024 & 5.330002 & -1.668625 \\ \mathrm{H} & 0.019272 & 5.000900 & -2.114410 \\ \mathrm{H} & 1.036469 & 2.790787 & -1.593216 \\ & & & \end{array}$

\section{$\left[\mathrm{L}^{8} \mathrm{py}_{2} \mathrm{Co}\left(\mathrm{MeS}-\mathrm{C}_{6} \mathrm{H}_{4}-p-\mathrm{CH}_{3}\right)\right]^{2+}\left(\mathbf{2}-\mathbf{C H}_{\mathbf{3}}\right)$}

$\begin{array}{lrrr}\text { Co } & 0.000000 & 0.000000 & 0.000000 \\ \mathrm{~S} & 0.000000 & 0.000000 & 2.453339 \\ \mathrm{C} & -1.627625 & -0.661469 & 2.841568 \\ \mathrm{C} & -2.763367 & 0.143326 & 3.000870 \\ \mathrm{C} & -3.990585 & -0.452911 & 3.292435 \\ \mathrm{C} & -4.120697 & -1.847534 & 3.413300 \\ \mathrm{C} & -2.964767 & -2.633957 & 3.248047 \\ \mathrm{C} & -1.725998 & -2.055664 & 2.975616 \\ \mathrm{C} & -5.446777 & -2.483322 & 3.740875 \\ \mathrm{C} & -0.067123 & 1.674042 & 3.192657 \\ \mathrm{H} & -2.707123 & 1.228180 & 2.917175 \\ \mathrm{H} & -4.866409 & 0.182526 & 3.435669 \\ \mathrm{H} & -3.028442 & -3.717331 & 3.365616 \\ \mathrm{H} & -0.835678 & -2.681137 & 2.897949 \\ \mathrm{H} & -5.413345 & -2.959518 & 4.731781 \\ \mathrm{H} & -5.700607 & -3.271759 & 3.018204 \\ \mathrm{H} & -6.258072 & -1.746643 & 3.749344 \\ \mathrm{H} & -0.340820 & 1.559494 & 4.248306 \\ \mathrm{H} & -0.784744 & 2.323334 & 2.680511 \\ \mathrm{H} & 0.942215 & 2.096634 & 3.125351 \\ \mathrm{~N} & 0.268219 & 1.992493 & -0.889496 \\ \mathrm{~N} & 2.243439 & -0.016571 & -0.202972 \\ \mathrm{~N} & -1.975311 & 0.375107 & -0.481827 \\ \mathrm{~N} & 0.461548 & -2.035339 & -0.358109 \\ \mathrm{C} & 1.194473 & 2.901016 & -0.132294\end{array}$




$\begin{array}{rrrr}\mathrm{C} & 2.680832 & 2.483383 & -0.112747 \\ \mathrm{C} & 2.967804 & 1.089645 & 0.488235 \\ \mathrm{C} & 2.561569 & -0.013306 & -1.682190 \\ \mathrm{C} & 1.370789 & 0.415985 & -2.587540 \\ \mathrm{C} & 0.718491 & 1.798447 & -2.320969 \\ \mathrm{C} & -1.085526 & 2.620148 & -0.847366 \\ \mathrm{C} & -2.219315 & 1.626038 & -0.946030 \\ \mathrm{C} & -3.489029 & 2.016251 & -1.377930 \\ \mathrm{C} & -4.541473 & 1.102188 & -1.320343 \\ \mathrm{C} & -4.293640 & -0.181931 & -0.828598 \\ \mathrm{C} & -3.005096 & -0.502487 & -0.418137 \\ \mathrm{C} & 2.647232 & -1.307266 & 0.406921 \\ \mathrm{C} & 1.730957 & -2.410751 & -0.056778 \\ \mathrm{C} & 2.155502 & -3.735550 & -0.175354 \\ \mathrm{C} & 1.256516 & -4.706665 & -0.618958 \\ \mathrm{C} & -0.044600 & -4.321457 & -0.953140 \\ \mathrm{C} & -0.395752 & -2.980453 & -0.815811 \\ \mathrm{H} & 0.814240 & 2.956894 & 0.898239 \\ \mathrm{H} & 1.126236 & 3.917618 & -0.563156 \\ \mathrm{H} & 3.217041 & 3.226242 & 0.499466 \\ \mathrm{H} & 3.117462 & 2.565704 & -1.119247 \\ \mathrm{H} & 4.058395 & 0.903549 & 0.446274 \\ \mathrm{H} & 2.676849 & 1.061920 & 1.550949 \\ \mathrm{H} & 2.839188 & -1.035065 & -1.980728 \\ \mathrm{H} & 3.445206 & 0.617691 & -1.867600 \\ \mathrm{H} & 0.585831 & -0.358994 & -2.531403 \\ \mathrm{H} & 1.722733 & 0.410980 & -3.632614 \\ \mathrm{H} & 1.394455 & 2.624924 & -2.599670 \\ \mathrm{H} & -0.168045 & 1.875473 & -2.968506 \\ \mathrm{H} & -1.180800 & 3.398529 & -1.620255 \\ \mathrm{H} & -1.181885 & 3.134766 & 0.121323 \\ \mathrm{H} & -3.651123 & 3.031815 & -1.742523 \\ \mathrm{H} & -5.541519 & 1.391006 & -1.645584 \\ \mathrm{H} & -5.086212 & -0.925583 & -0.754730 \\ \mathrm{H} & -2.779938 & -1.482269 & 0.002289 \\ \mathrm{H} & 2.575958 & -1.205582 & 1.501953 \\ \mathrm{H} & 3.696808 & -1.554962 & 0.171890 \\ \mathrm{H} & 3.187012 & -3.996887 & 0.067764 \\ \mathrm{H} & 1.573914 & -5.745224 & -0.723312 \\ \mathrm{H} & -0.771439 & -5.036285 & -1.337204 \\ \mathrm{H} & -1.388153 & -2.634323 & -1.096405 \\ & & & \end{array}$

\section{$\left[\mathrm{L}^{8} \mathrm{py}_{2} \mathrm{Ni}\left(\mathrm{MeS}-\mathrm{C}_{6} \mathrm{H}_{4}-p-\mathrm{CH}_{3}\right)\right]^{2+}\left(\mathbf{3}-\mathbf{C H}_{\mathbf{3}}\right)$}

$\begin{array}{lrrr}\mathrm{Ni} & 0.000000 & 0.000000 & 0.000000 \\ \mathrm{~S} & 0.000000 & 0.000000 & 2.419708 \\ \mathrm{C} & 1.211487 & 1.181015 & 3.009247 \\ \mathrm{C} & 2.428040 & 0.664886 & 3.474731 \\ \mathrm{C} & 3.422546 & 1.539764 & 3.913040 \\ \mathrm{C} & 3.227615 & 2.930313 & 3.905548 \\ \mathrm{C} & 1.983627 & 3.424026 & 3.459213 \\ \mathrm{C} & 0.984161 & 2.567642 & 3.006226 \\ \mathrm{C} & 4.308548 & 3.870895 & 4.367950 \\ \mathrm{C} & -1.519470 & 0.603134 & 3.252686 \\ \mathrm{H} & 2.589676 & -0.412811 & 3.512146 \\ \mathrm{H} & 4.364639 & 1.130814 & 4.281036\end{array}$

- S8 - 


\begin{tabular}{rrrr}
$\mathrm{H}$ & 1.799133 & 4.500015 & 3.465057 \\
$\mathrm{H}$ & 0.037445 & 2.980713 & 2.657425 \\
$\mathrm{H}$ & 4.556931 & 4.601791 & 3.584335 \\
$\mathrm{H}$ & 5.223282 & 3.331223 & 4.637848 \\
$\mathrm{H}$ & 3.979889 & 4.445953 & 5.245575 \\
$\mathrm{H}$ & -1.314514 & 0.613525 & 4.329300 \\
$\mathrm{H}$ & -2.322769 & -0.112991 & 3.045975 \\
$\mathrm{H}$ & -1.799240 & 1.604950 & 2.911453 \\
$\mathrm{~N}$ & 0.302200 & -2.076385 & -0.480316 \\
$\mathrm{~N}$ & -2.040436 & -0.337143 & -0.511398 \\
$\mathrm{~N}$ & 2.097229 & -0.020935 & -0.027100 \\
$\mathrm{~N}$ & -0.577042 & 1.955872 & -0.516754 \\
$\mathrm{C}$ & -0.616745 & -3.022980 & 0.228119 \\
$\mathrm{C}$ & -2.110474 & -2.862198 & -0.118057 \\
$\mathrm{C}$ & -2.714508 & -1.475403 & 0.189758 \\
$\mathrm{C}$ & -2.030029 & -0.565338 & -2.007584 \\
$\mathrm{C}$ & -0.618729 & -0.903931 & -2.571182 \\
$\mathrm{C}$ & 0.130386 & -2.132919 & -1.982880 \\
$\mathrm{C}$ & 1.695343 & -2.425293 & -0.098862 \\
$\mathrm{C}$ & 2.639160 & -1.252441 & -0.197800 \\
$\mathrm{C}$ & 4.016281 & -1.444977 & -0.334854 \\
$\mathrm{C}$ & 4.871307 & -0.345413 & -0.272842 \\
$\mathrm{C}$ & 4.320053 & 0.919312 & -0.060272 \\
$\mathrm{C}$ & 2.939621 & 1.034332 & 0.061203 \\
$\mathrm{C}$ & -2.755966 & 0.922607 & -0.176407 \\
$\mathrm{C}$ & -1.922928 & 2.130280 & -0.534286 \\
$\mathrm{C}$ & -2.508713 & 3.355408 & -0.861252 \\
$\mathrm{C}$ & -1.693253 & 4.435913 & -1.199081 \\
$\mathrm{C}$ & -0.310532 & 4.245529 & -1.231384 \\
$\mathrm{C}$ & 0.200516 & 2.998322 & -0.888565 \\
$\mathrm{H}$ & -0.464020 & -2.869797 & 1.308273 \\
$\mathrm{H}$ & -0.315109 & -4.061218 & -0.005569 \\
$\mathrm{H}$ & -2.664703 & -3.598145 & 0.486160 \\
$\mathrm{H}$ & -2.299255 & -3.141983 & -1.165268 \\
$\mathrm{H}$ & -3.786179 & -1.485031 & -0.085312 \\
$\mathrm{H}$ & 1.270370 & 2.814102 & -0.932159 \\
$\mathrm{H}$ & -2.656616 & -1.276596 & 1.270782 \\
$\mathrm{H}$ & -2.362473 & 0.360504 & -2.500015 \\
$\mathrm{H}$ & -2.761856 & -1.347000 & -2.265839 \\
$\mathrm{H}$ & 0.028305 & -0.011963 & -2.463165 \\
$\mathrm{H}$ & -0.718033 & -1.061966 & -3.658142 \\
$\mathrm{H}$ & -0.367630 & -3.077774 & -2.257874 \\
$\mathrm{H}$ & 1.132019 & -2.151993 & -2.438553 \\
$\mathrm{H}$ & 2.066605 & -3.278564 & -0.688370 \\
$\mathrm{H}$ & 1.680511 & -2.752670 & 0.952408 \\
$\mathrm{H}$ & 4.411285 & -2.451981 & -0.475388 \\
$\mathrm{H}$ & 5.949249 & -0.475479 & -0.374222 \\
$\mathrm{H}$ & 2.946579 & 1.807297 & 0.018265 \\
$\mathrm{H}$ & -2.956024 & 1.999237 & 0.271469 \\
0.917404 & 0.905411 \\
\hline
\end{tabular}




\section{$\left[\mathrm{L}^{8} \mathrm{py}_{2} \mathrm{Zn}\left(\mathrm{MeS}-\mathrm{C}_{6} \mathrm{H}_{4}-p-\mathrm{CH}_{3}\right)\right]^{2+}\left(\mathbf{4}-\mathbf{C H}_{3}\right)$}

\begin{tabular}{|c|c|c|c|}
\hline $\mathrm{Zn}$ & 0.000000 & 0.000000 & 0.000000 \\
\hline S & 0.000000 & 0.000000 & 2.505493 \\
\hline C & -1.151947 & 1.317764 & 2.926956 \\
\hline$C$ & -0.594681 & 2.513992 & 3.387207 \\
\hline C & -1.431168 & 3.599640 & 3.656097 \\
\hline C & -2.821976 & 3.505157 & 3.498337 \\
\hline C & -3.362030 & 2.273911 & 3.071304 \\
\hline C & -2.541153 & 1.188248 & 2.772568 \\
\hline C & -3.715775 & 4.681885 & 3.793365 \\
\hline C & -0.700165 & -1.453232 & 3.384689 \\
\hline $\mathrm{H}$ & 0.481750 & 2.596497 & 3.539932 \\
\hline $\mathrm{H}$ & -0.990112 & 4.533386 & 4.008591 \\
\hline $\mathrm{H}$ & -4.443222 & 2.170044 & 2.961670 \\
\hline $\mathrm{H}$ & -2.982986 & 0.252900 & 2.426956 \\
\hline $\mathrm{H}$ & -4.395905 & 4.885559 & 2.954300 \\
\hline $\mathrm{H}$ & -3.132233 & 5.588196 & 3.992523 \\
\hline $\mathrm{H}$ & -4.343094 & 4.484116 & 4.674423 \\
\hline $\mathrm{H}$ & -0.703766 & -1.210678 & 4.453369 \\
\hline $\mathrm{H}$ & -0.029907 & -2.303300 & 3.209839 \\
\hline $\mathrm{H}$ & -1.713226 & -1.685654 & 3.042450 \\
\hline $\mathrm{N}$ & 2.145630 & 0.097733 & -0.616348 \\
\hline $\mathrm{N}$ & 0.228653 & -2.106216 & -0.608170 \\
\hline $\mathrm{N}$ & 0.230331 & 2.114334 & -0.219742 \\
\hline $\mathrm{N}$ & -1.959579 & -0.458023 & -0.794083 \\
\hline C & 2.989807 & -0.861526 & 0.172043 \\
\hline C & 2.720108 & -2.354080 & -0.109970 \\
\hline C & 1.278870 & -2.841400 & 0.166916 \\
\hline C & 0.518494 & -2.175064 & -2.092148 \\
\hline C & 0.982483 & -0.824554 & -2.708313 \\
\hline C & 2.240402 & -0.145081 & -2.104584 \\
\hline C & 2.593063 & 1.474350 & -0.284744 \\
\hline C & 1.503525 & 2.514236 & -0.446000 \\
\hline C & 1.817947 & 3.851318 & -0.700546 \\
\hline $\mathrm{C}$ & 0.800629 & 4.805420 & -0.703888 \\
\hline C & -0.507721 & 4.396927 & -0.435791 \\
\hline C & -0.748337 & 3.049118 & -0.195862 \\
\hline C & -1.101105 & -2.695526 & -0.314972 \\
\hline C & -2.214691 & -1.789215 & -0.800293 \\
\hline C & -3.441086 & -2.301743 & -1.228470 \\
\hline C & -4.431320 & -1.424759 & -1.673553 \\
\hline C & -4.157120 & -0.055634 & -1.696655 \\
\hline C & -2.914612 & 0.383255 & -1.251358 \\
\hline $\mathrm{H}$ & 2.812988 & -0.645752 & 1.238358 \\
\hline $\mathrm{H}$ & 4.057617 & -0.657196 & -0.031738 \\
\hline $\mathrm{H}$ & 3.389816 & -2.932907 & 0.546158 \\
\hline $\mathrm{H}$ & 3.021942 & -2.612823 & -1.135864 \\
\hline $\mathrm{H}$ & 1.218460 & -3.921890 & -0.063950 \\
\hline $\mathrm{H}$ & 1.044266 & -2.722855 & 1.236908 \\
\hline $\mathrm{H}$ & -0.405228 & -2.469208 & -2.612564 \\
\hline $\mathrm{H}$ & 1.254456 & -2.972321 & -2.282500 \\
\hline $\mathrm{H}$ & 0.141815 & -0.109192 & -2.659775 \\
\hline $\mathrm{H}$ & 1.174164 & -0.991730 & -3.780823 \\
\hline
\end{tabular}




$\begin{array}{rrrr}\mathrm{H} & 3.155441 & -0.722519 & -2.320511 \\ \mathrm{H} & 2.355484 & 0.830292 & -2.601944 \\ \mathrm{H} & 3.482208 & 1.759674 & -0.868347 \\ \mathrm{H} & 2.897446 & 1.483505 & 0.773926 \\ \mathrm{H} & 2.854050 & 4.139114 & -0.883453 \\ \mathrm{H} & 1.027405 & 5.853394 & -0.902679 \\ \mathrm{H} & -1.331238 & 5.109543 & -0.408585 \\ \mathrm{H} & -1.748428 & 2.697372 & 0.051697 \\ \mathrm{H} & -1.181427 & -2.825165 & 0.776184 \\ \mathrm{H} & -1.209778 & -3.696548 & -0.764908 \\ \mathrm{H} & -3.610504 & -3.379562 & -1.223709 \\ \mathrm{H} & -5.393829 & -1.806488 & -2.015854 \\ \mathrm{H} & -4.888397 & 0.664200 & -2.063492 \\ \mathrm{H} & -2.659485 & 1.440140 & -1.276764\end{array}$


Figure S1. Cyclic voltammogram of $5,\left[\mathrm{~L}^{8} \mathrm{py}_{2} \mathrm{Co}\left(\mathrm{CH}_{3} \mathrm{CN}\right)\right]^{2+}$

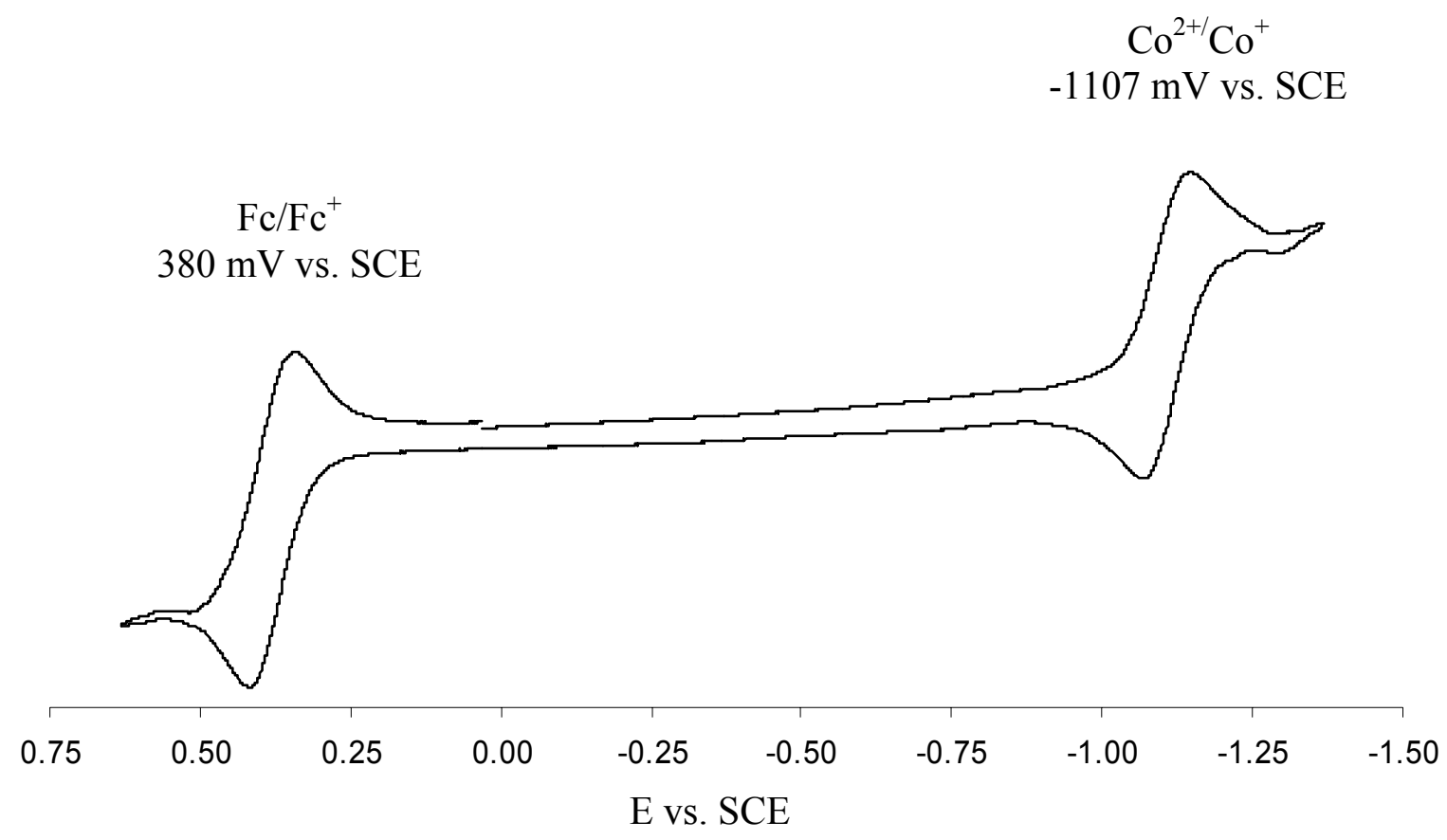

Figure S2. Cyclic voltammogram of $\mathbf{6},\left[\mathrm{L}^{8} \mathrm{py}_{2} \mathrm{Ni}\left(\mathrm{CH}_{3} \mathrm{CN}\right)\right]^{2+}$

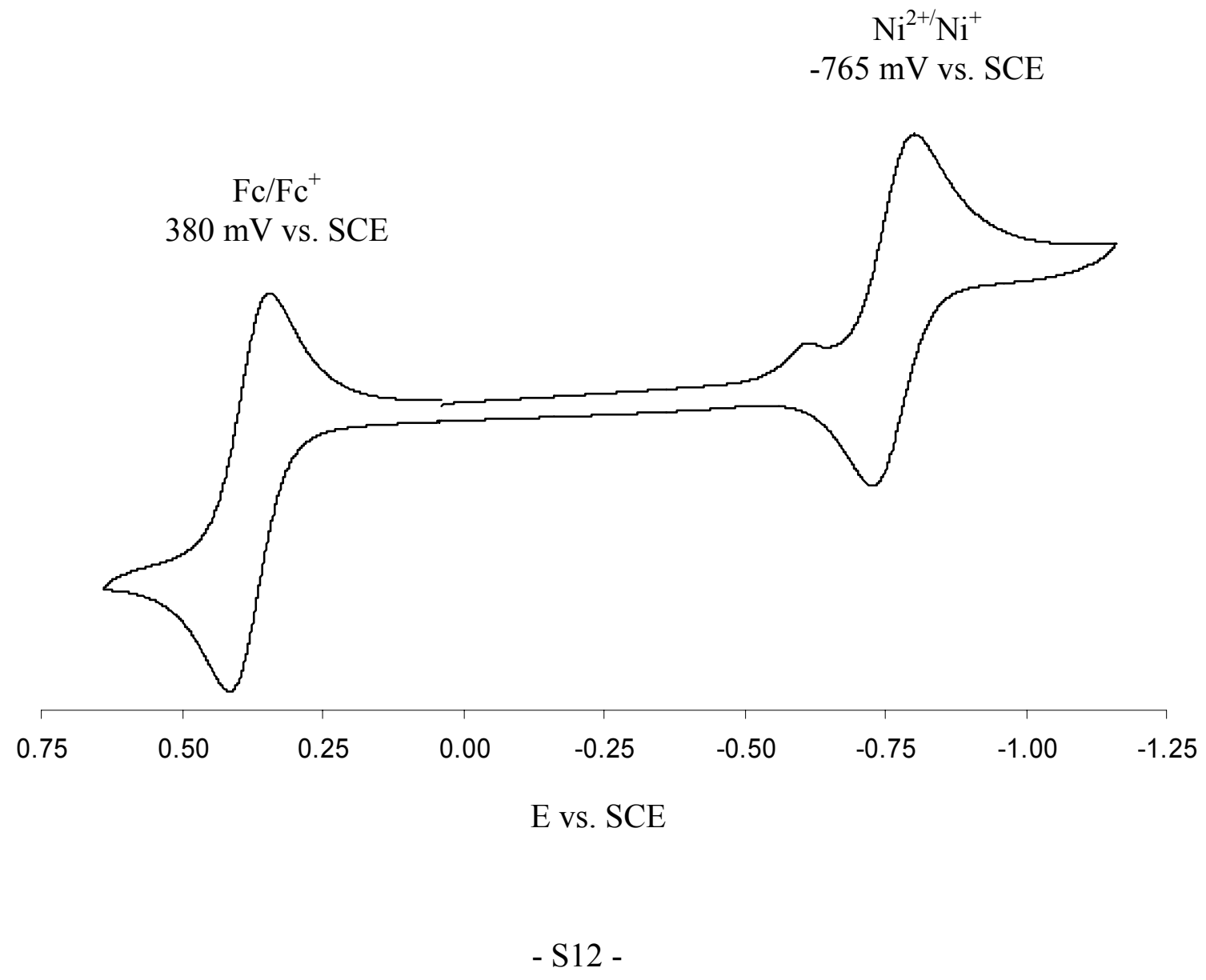


Figure S3. Cyclic voltammogram of 2, $\left[\mathrm{L}^{8} \mathrm{py}_{2} \mathrm{Co}(\mathrm{SAr})\right]^{+}$

(Referenced to external ferrocene due to the proximity of the $\mathrm{Fc} / \mathrm{Fc}^{+}$couple to that of $\mathrm{Co}^{2+} / \mathrm{Co}^{3+}$ )

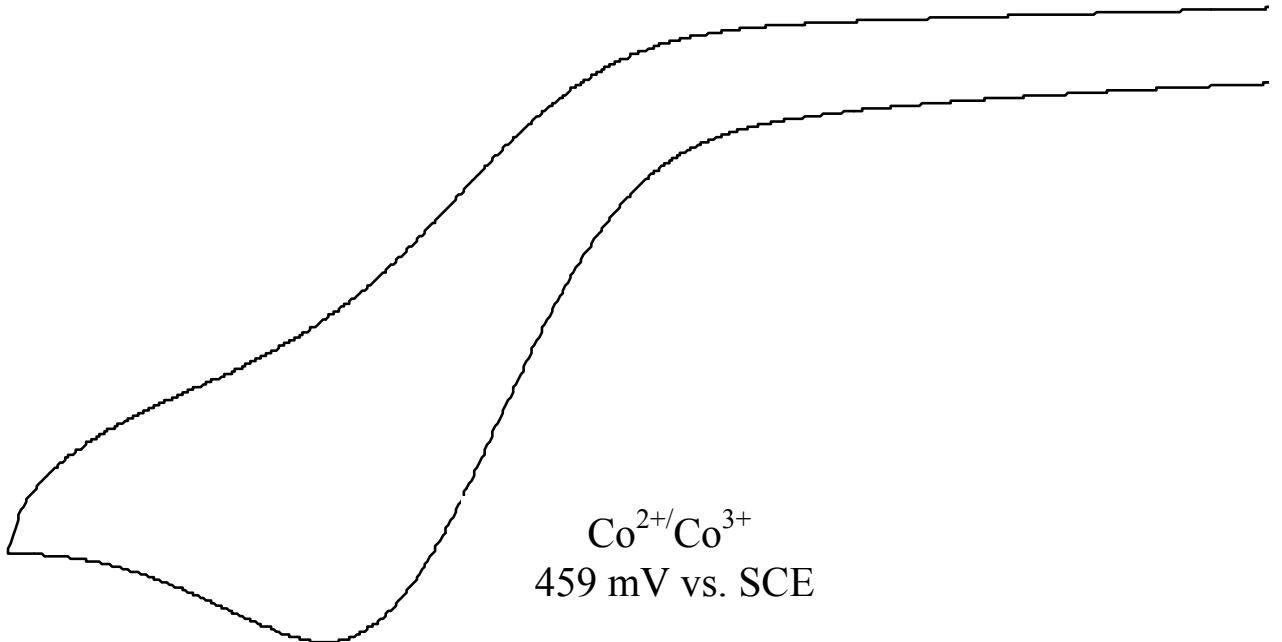

\begin{tabular}{|c|c|c|c|c|c|c|c|}
\hline 0.70 & 0.60 & 0.50 & 0.40 & 0.30 & 0.20 & 0.10 & 0.00 \\
\hline
\end{tabular}

Figure S4. Cyclic voltammogram of 3, $\left[\mathrm{L}^{8} \mathrm{py}_{2} \mathrm{Ni}(\mathrm{SAr})\right]^{+}$

(Referenced to external ferrocene due to the proximity of the $\mathrm{Fc} / \mathrm{Fc}^{+}$couple to that of $\mathrm{Ni}^{2+} / \mathrm{Ni}^{3+}$ )

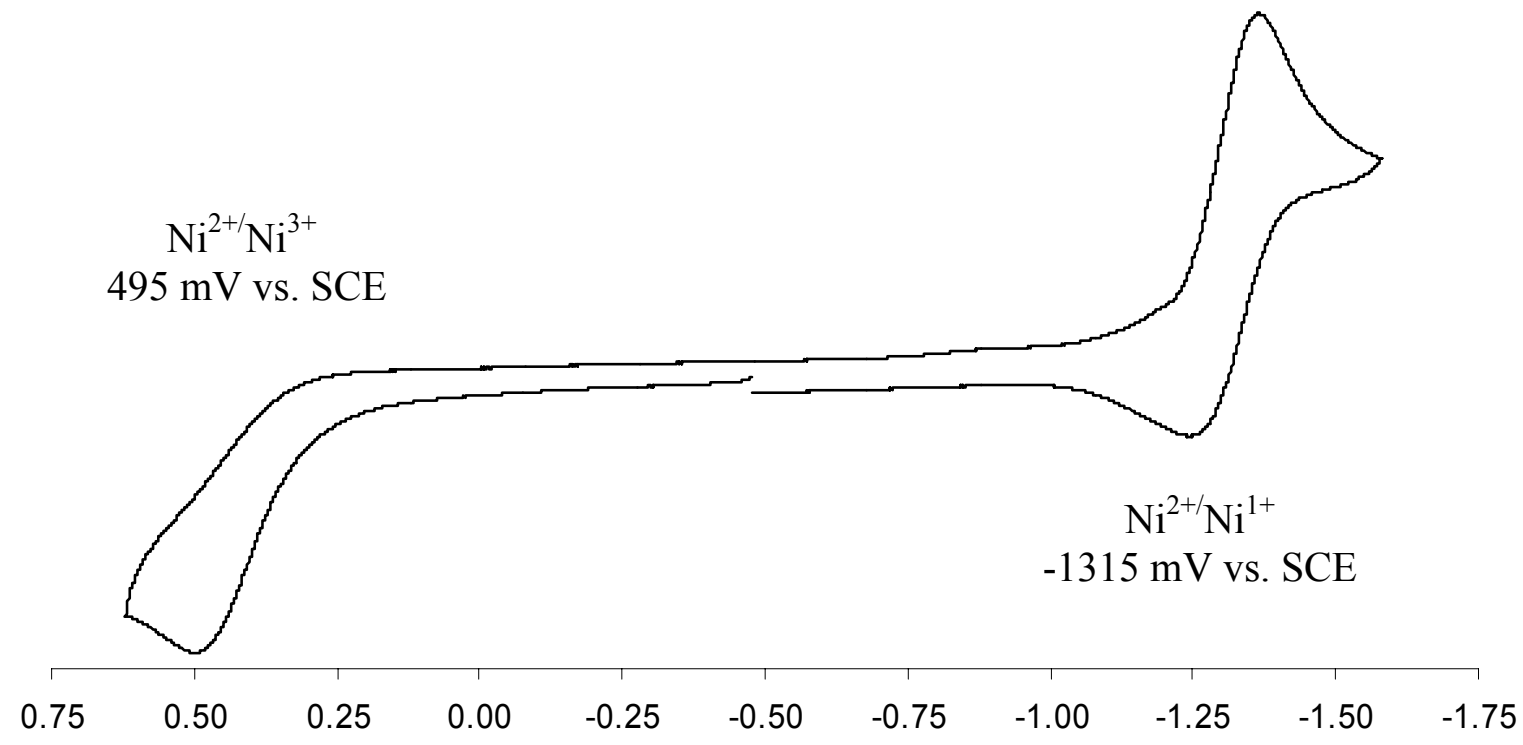

E vs. SCE 